\title{
Continuous energy measurement of the electron beam in the storage ring of Diamond Light Source with resonant spin depolarization
}

\author{
Niki Vitoratou®* \\ John Adams Institute at Royal Holloway, Egham, Surrey TW20 OEX, United Kingdom, \\ and Diamond Light Source Ltd., Harwell Science and Innovation Campus, \\ Didcot, OX11 ODE, United Kingdom \\ Pavel Karataev \\ John Adams Institute at Royal Holloway, Egham, Surrey TW20 OEX, United Kingdom \\ Guenther Rehm $\odot$ \\ Diamond Light Source Ltd., Harwell Science and Innovation Campus, \\ Didcot, OX11 ODE, United Kingdom
}

(Received 20 August 2019; published 10 December 2019)

\begin{abstract}
Electron spin polarization occurs naturally in an electron storage ring due to spin-flip synchrotron radiation. The equilibrium polarization level that is achieved in Diamond Light Source was studied theoretically and experimentally. A novel approach that enables measurements during user operation is presented, compatible with beams of small vertical emittance in the range of pm rad. An array of four custom-made scintillation detectors has been installed around the beam pipe downstream from the collimators to capture a significant fraction of the lost particles and witness the depolarization process in the presence of a background loss rate. A new concept for detecting the depolarization resonance even when it coincides with higher-order betatron resonances was introduced. The momentum compaction factor was measured to be in very good agreement with the storage ring numerical model. In the end, we measured a good long-term stability of the beam energy during user operation time.
\end{abstract}

DOI: 10.1103/PhysRevAccelBeams.22.122801

\section{INTRODUCTION}

Resonant spin depolarization (RSD) is a wellestablished technique to measure the electron beam energy in synchrotrons [1-5]. The spin of the electrons is aligned antiparallel with the magnetic field of bending magnets due to spin-flip synchrotron radiation. Using a device that can generate a time-varying horizontal magnetic field, Ithe spin vector can be resonantly tilted away from the vertical axis by a small amount in successive revolutions of the storage ring, resulting gradually in the reduction of the beam polarization. The depolarization leads to an increase of Touschek scattering and, consequently, in the stored beam loss rate. The increase in beam losses is used to determine the precession frequency of the electrons, and, thus, the energy can be calculated.

\footnotetext{
Niki.Vitoratou.2016@live.rhul.ac.uk
}

Published by the American Physical Society under the terms of the Creative Commons Attribution 4.0 International license. Further distribution of this work must maintain attribution to the author(s) and the published article's title, journal citation, and DOI.
In the Diamond Light Source storage ring, the electron beam consists of up to 936 bunches with a total current of $300 \mathrm{~mA}$, a vertical emittance of $8 \mathrm{pm}$ rad, and an energy of $3.0145 \mathrm{GeV}$. Energy measurements with RSD have been achieved [6] but so far have not been compatible with operational user time and required long time periods during the dedicated machine development. This resulted in rare readings of the electron beam energy. The implementation of a technique that makes RSD compatible with user time would give information about the energy stability of the electron beam.

In this paper, the details of the experimental setup used for these measurements are presented. A range of different parameters in order to accomplish the energy measurements with high accuracy and repeatability during user time have been studied.

\section{THEORY}

\section{A. Beam polarization}

According to the Sokolov-Ternov effect, the spin of the electron beam will develop a polarization antiparallel with the magnetic field of the main bending magnets. This is a result of the quantum emission of synchrotron radiation, 
which causes the spin state to flip either parallel or antiparallel to the magnetic field of the main bending magnets with a transition rate which is asymmetric and in favor of the antiparallel direction for electrons. This asymmetry, in an ideal ring in the absence of depolarizing effects, leads to a maximum beam polarization of $92 \%$ [7]. The polarization of the beam, for the case of Diamond Light Source (DLS), was studied theoretically and was verified experimentally.

\section{B. Beam depolarization}

The spin precession frequency of an ultrarelativistic electron in a magnetic field follows the ThomasBargmann-Michel-Telegdi equation which, for a light source storage ring where there are no significant solenoid magnetic fields nor transverse electric fields, can be simplified to the form below [8]:

$$
\Omega_{z}=\omega_{0}\left(1+\alpha_{g} \gamma\right),
$$

where $\omega_{0}$ is the revolution frequency, $\alpha_{g}=(g-2) / 2 \simeq$ 0.0011597 the anomalous gyromagnetic ratio, and $\gamma$ the relativistic factor. The product $\alpha_{g} \gamma$ is the number of revolutions the spin vector makes about the vertical axis in one revolution of the storage ring defined as the spin tune $\nu$. The relationship between the spin tune and the energy is the basis of the energy measurement, as by measuring the spin tune the energy can be extracted.

For the determination of the spin tune, the stored polarized beam is excited by a horizontal magnetic field produced by a pair of vertical strip lines. The magnetic field is swept across frequencies $\left(f_{\text {dep }}\right)$ in an attempt to match the fractional part of the spin tune:

$$
f_{\text {dep }}=\left(\alpha_{g} \gamma+k\right) \cdot f_{\text {rev }},
$$

where $k$ is an integer and $f_{\text {rev }}$ the revolution frequency. When the above relationship is valid, the spin vector gradually starts to tilt away from the vertical axis as a result of the resonance, and the beam gets depolarized. The decrease of polarization leads to an increase of the beam losses. The beam losses are used to identify the resonant depolarization of the beam and, consequently, indicate the spin tune and the beam energy.

\section{Froissart-Stora equation}

A vertically polarized beam can be depolarized by a perturbing horizontal magnetic field when the perturbation frequency matches the spin resonance. The Froissart-Stora formula describes the spin transport through a single resonance where the final polarization is given by [9]

$$
P_{y}(\infty)=\left(2 e^{\pi|\epsilon|^{2} / 2 \alpha_{r}}-1\right) P_{y}(-\infty),
$$

where $\epsilon$ is the resonance strength and $\alpha_{r}$ the rate of resonance crossing. This ratio is a function of the frequency step $\Delta f$, the excitation time $\Delta t$, and the revolution frequency $f_{0}$ by the equation $\alpha_{r}=\frac{\Delta f_{r f}}{2 \pi f_{0}^{2} \Delta t} . P_{y}(\infty)$ and $P_{y}(-\infty)$ refer to the initial and final polarization, respectively. This formula shows that a large polarization loss can occur when the crossing speed is comparable to the square of the resonance strength. Because this calculation is valid only for a single particle without the effect of synchrotron radiation, it cannot give exact results of the final polarization in our case. However, this relationship is useful to understand what parameters we should adjust in order to maximize the depolarization level of the beam through a scan.

\section{POLARIZATION STUDIES}

\section{A. Polarization experimental study}

The polarization of the beam can be observed experimentally by monitoring the beam lifetime. The relation between Touschek lifetime $\tau$, Touschek beam losses $d N / d t$, and polarization $P$ is shown [10]:

$$
\frac{1}{\tau}=-\frac{1}{N} \frac{d N}{d t}=\alpha_{t}\left[C(\varepsilon)+F(\varepsilon) P^{2}\right] N,
$$

where

$$
\begin{gathered}
C(\varepsilon)=\varepsilon \int_{\varepsilon}^{\infty} \frac{1}{u^{2}}\left\{\left(\frac{u}{\varepsilon}\right)-\frac{1}{2} \ln \left(\frac{u}{\varepsilon}\right)-1\right\} e^{-u} d u, \\
F(\varepsilon)=-\frac{\varepsilon}{2} \int_{\varepsilon}^{\infty} \frac{1}{u^{2}} \ln \frac{u}{\varepsilon} e^{-u} d u,
\end{gathered}
$$

and

$$
\varepsilon=\left(\frac{\delta_{\mathrm{acc}}}{\gamma} \frac{\beta_{x}}{\sigma_{x}}\right)^{2}
$$

where $\delta_{\text {acc }}$ is the momentum acceptance, $\gamma$ is the Lorentz relativistic factor, $\sigma_{x}$ is the horizontal beam size, and $\beta_{x}$ is the horizontal beta function. The coefficient $\alpha_{t}$ is inversely proportional to the electron bunch volume $V=\sigma_{x} \sigma_{y} \sigma_{s}$. For a measurement, these numerical integrals can be treated as constants and have been evaluated using Mathematica [11].

The relative increase of the lifetime due to the polarization is given by the following relation:

$$
\frac{\tau_{(0)}}{\tau_{(S)}}=1+\frac{F(\varepsilon)}{C(\varepsilon)} P^{2},
$$

where $\tau_{(0)}$ corresponds to the initial lifetime after the injection and $\tau_{(S)}$ is the lifetime value during the polarization. Using Eq. (8), we can have an estimation of the polarization level during the polarization process.

The amount of polarization will be a combination of the polarizing and depolarizing effects by horizontal magnetic fields due to closed orbit distortions or quadrupole misalignments. It is given by [12] 


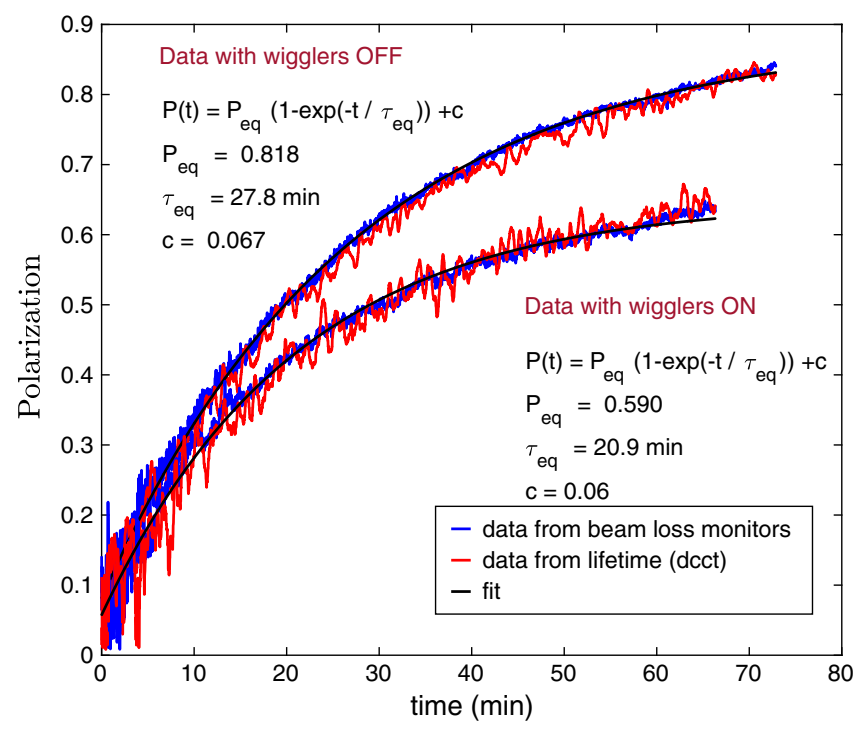

FIG. 1. Beam polarization as a function of time measured for wigglers off and on. A fit according to Eq. (9) calculates the maximum polarization level and the polarization time.

$P(t)=P_{\mathrm{ST}} \frac{\tau_{d}}{\tau_{d}+\tau_{\mathrm{ST}}}\left[1-\exp \left(-\frac{t}{\tau_{\mathrm{ST}}}\left(\frac{\tau_{d}+\tau_{\mathrm{ST}}}{\tau_{d}}\right)\right)\right]$,

where $P_{\mathrm{ST}}$ and $\tau_{\mathrm{ST}}$ are the Sokolov-Ternov values for the equilibrium polarization level and the time constant, respectively, and $\tau_{d}$ is the depolarization time constant.

The equilibrium polarization level $P_{\mathrm{ST}}$ is related with the integral of the magnetic fields $B$ along the curvilinear trajectory of the beam $s$ [5]:

$$
P_{\mathrm{ST}}=\frac{8}{5 \sqrt{3}} \frac{\oint B^{3} d s}{\oint|B|^{3} d s}
$$

which for an isomagnetic planar ring equals to the maximum value of $92 \%$. When wiggler insertion devices, of which there exist two electrically powered devices in DLS, are introduced, the polarization level is reduced.

In Fig. 1, the polarization built up with both wigglers turned off and on is displayed from a recording of the beam lifetime plotted versus time. A fit according to Eq. (9) calculates the maximum polarization level and the polarization time. An offset was introduced to include the polarization that had already been present at the beginning of the data acquisition. The beam lifetime can be derived by fitting a slope on the measurement of the beam current using a parametric current transformer. Alternatively, a beam lifetime proportional value can be calculated from the measurement of beam losses and the beam current using Eq. (4). The second approach, as is shown in Fig. 1, results in less noisy data.

Because of the excellent alignment given by orbit correction schemes and resulting in the small vertical emittance in DLS, the reduction of equilibrium polarization is very small, allowing the beam to acquire a polarization level of $88 \%$ with

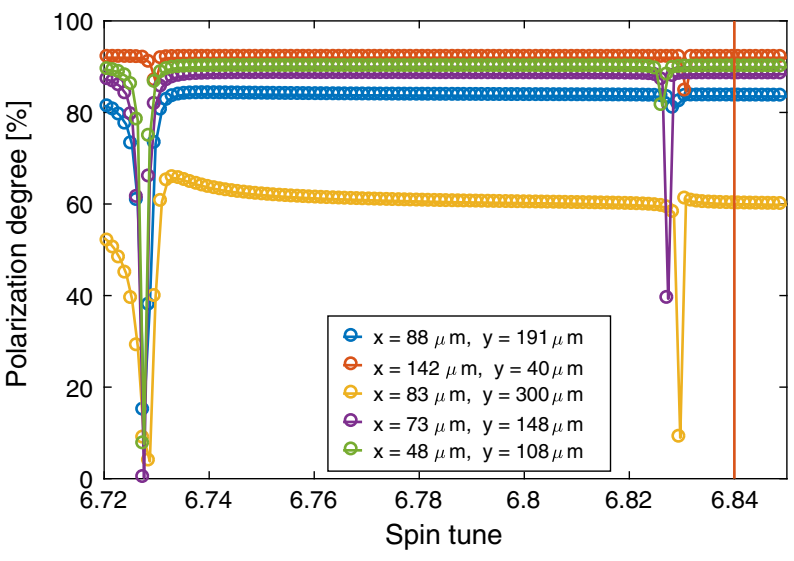

FIG. 2. The beam polarization level calculated by SLIM for various sets of closed orbit distortions which were generated randomly by the orbit disturbances. The rms values of the horizontal and vertical orbit distortions are shown in the legend. The red vertical line shows the experimental operational spin tune.

a polarization time of $27 \mathrm{~min}$. When the wigglers are turned on, the maximum beam polarization is reduced according to Eq. (10). From experimental data, we found that the maximum polarization for this case was $65 \%$.

\section{B. Simulation study of polarization}

Imperfections or intrinsic resonances can arise due to magnet field and alignment errors or betatron oscillations, respectively, and can limit the maximum level of polarization. A high initial level of polarization of the beam is critical for the implementation of the energy measurements with RSD. To ensure that the beam polarizes in the case of DLS, the beam polarization level was calculated using the numerical simulation code SLIM [13]. A thin lens approximation of the diamond lattice was used as an input. A random distribution of vertical orbit distortions was introduced to the model in order to simulate the field imperfections. The strength of a quadrupole doublet was adjusted to keep the betatron tunes in agreement with the model after the introduction of the closed orbit distortions (CODs) from the field errors. The horizontal CODs result from the coupling with the vertical CODs. In Fig. 2, the right-hand resonances show that when the spin tune $\nu$ and the horizontal betatron tune $\nu_{x}$ meet the condition $\nu+\nu_{x}=35$ the polarization degree can be reduced. When the spin tune is close to the intrinsic resonance $\nu+\nu_{y}=20$, where $\nu_{y}$ is the vertical betatron tune, the beam can be depolarized strongly, as is illustrated by the left-side resonances in Fig. 2. The betatron, synchrotron, and spin tunes of the diamond ring are listed in Table I. Different simulated field imperfections give different results of the position and the width of the resonances. To quantify the simulated field imperfection, the rms value of the closed orbit distortion that is introduced is calculated. Since the spin depolarization resonances do not diverge strongly and are far from the operational spin tune (6.84 for 
TABLE I. The diamond storage ring parameters.

\begin{tabular}{lcc}
\hline \hline Parameter & Values & Units \\
\hline Energy & 3.014 & $\mathrm{GeV}$ \\
Horizontal betatron tune $\nu_{x}$ & 28.189 & $\ldots$ \\
Vertical betatron tune $\nu_{y}$ & 13.277 & $\ldots$ \\
Synchrotron tune $\nu_{s}$ & 0.0041 & $\ldots$ \\
Spin tune $\nu$ & 6.840 & $\ldots$ \\
Theoretical polarization time $\tau_{\mathrm{ST}}$ & 29.9 & $\mathrm{~min}$ \\
\hline \hline
\end{tabular}

the DLS case), a high level of beam polarization during operation can be expected. Only when the CODs are large (in the range of hundreds of microns, much more than we anticipate in the case of DLS) is the equilibrium polarization reduced away from the resonances. These simulations results revealed that attention should be given to good orbit correction and that high-quality alignment of the magnets is mandatory. In addition, the betatron tunes should be chosen carefully in order to avoid any destructive spin depolarization resonances.

\section{EXPERIMENTAL SETUP}

\section{A. Excitation scheme}

The experimental setup consists of the excitation and the detection scheme. For the excitation, we use two $30-\mathrm{cm}-$ long vertical kicker strip lines [14] which are a part of the transverse multibunch feedback (TMBF) and can generate a horizontal oscillating magnetic field with an integrated strength of up to $8 \mu \mathrm{Tm}$. A numerically controlled oscillator, which is integrated into the feedback system, can be modulated with an internally synchronized rectangular waveform to generate a magnetic field which excites only a selected part of the beam. In Fig. 3(a), a typical fill pattern of the storage ring is shown. It consists of $936 \mathrm{rf}$ buckets, of which 900 are populated with around $500 \mathrm{pC}$ and 36 are deliberately unpopulated. The depolarization (carrier) signal, which is a sinusoidal signal with a frequency close to the spin tune, and the modulation (message) signal, which is a rectangular signal with a period which equals the revolution time, are illustrated in plot Figs. 3(b) and 3(c), respectively. The modulated signal in Fig. 3(d) will excite only the selected bunches of the fill pattern, leaving the others unaffected. The part of the beam which is excited can be set by adjusting the duty cycle of the message signal. This feature is essential for the implementation of the online energy measurements, where the data from the excited and the nonexcited part of the beam are used to detect the depolarization event excluding the background data from external factors.

\section{B. Detection scheme}

The detection scheme consists of a set of four detectors connected with a commercial acquisition instrument. Four blocks, $15 \mathrm{~cm}$ long, made of scintillator EJ204 [15], as is shown in Fig. 4, were manufactured in order to fit around
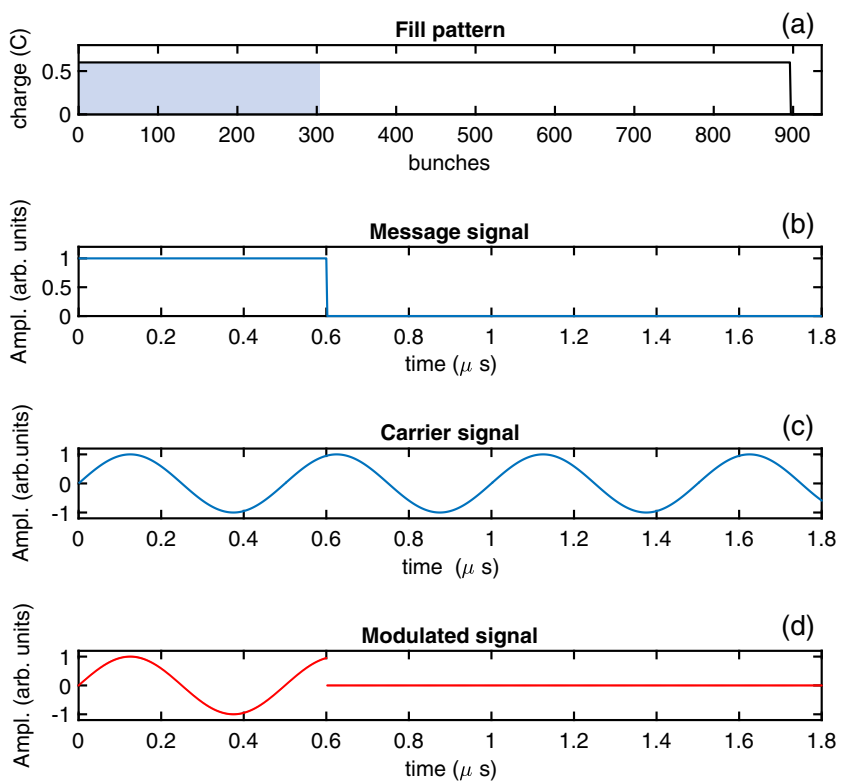

FIG. 3. The excitation regime for the online measurements includes a sinusoidal signal in frequencies according to the spin tune (c) and a rectangular waveform which modulates the signal (b). The modulated signal (d) excites only one part of the fill pattern (colored light blue), leaving the rest unaffected.

the octagonal shape of the beam pipe. They were wrapped in aluminum foil to enhance the reflection of the produced light and black taped for protection from external light. The design of the detectors was chosen based on the results from radiochromic film RTQA2 [16] that was installed in the same area as the detectors [17]. The radiochromic film which covers an area of $3 \mathrm{~cm} \times 3 \mathrm{~cm}$ was installed in the storage ring and is shown on the left in Fig. 5. The film was exposed for $8 \mathrm{~h}$ in the radiation produced by the electron beam under user operation conditions, where the beam current is $300 \mathrm{~mA}$ and the beam lifetime $11 \mathrm{~h}$. The darkening of the film was expressed as a dose as shown on the right in Fig. 5 using a calibration formula. The highest dose revealed

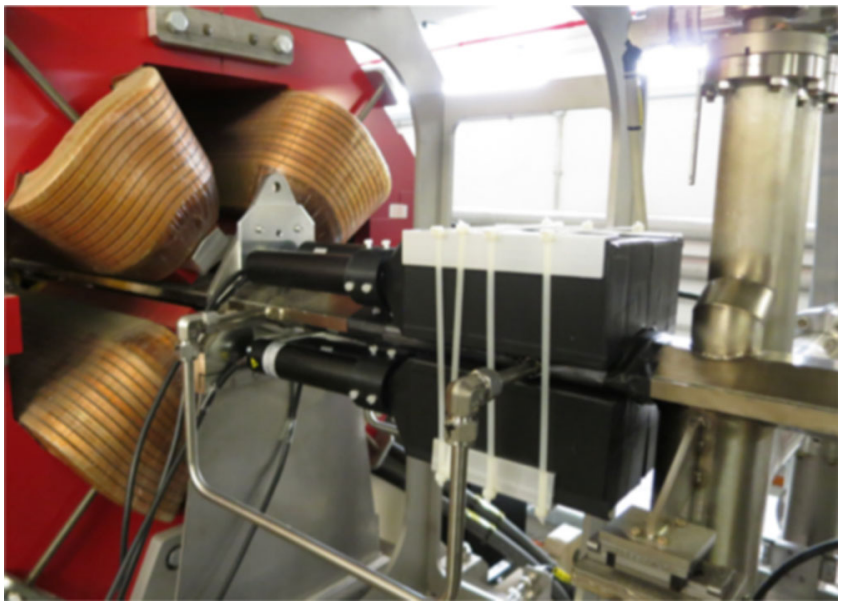

FIG. 4. The detector setup. 

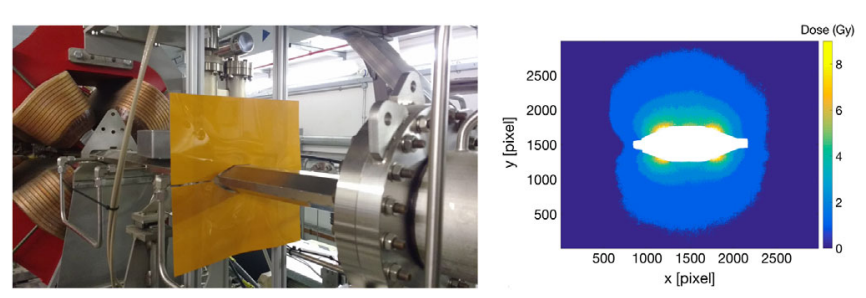

FIG. 5. The radiochromic film installed in the storage ring of DLS (left) and the calculated dose (right).

the area where the highest fraction of shower events caused by beam losses occur. The detectors should be positioned such as to detect the maximum number of Touschek particles. Touschek particles transfer high momentum from transverse to longitudinal motion, exceed the longitudinal acceptance limit, and are lost in areas with high dispersion and limited physical aperture. The area downstream from the collimators was chosen, as this is the location with the largest ratio of horizontal dispersion to physical aperture.

The four detectors were equipped with photomultipliers with a bialkali photocathode that matches with the wavelength of the scintillator light. Thin silicone pads were placed between the photocathode and the ends of the scintillator block in order to avoid any reflections when the light passes through two different materials. The monitors are protected from background ionizing radiation by a lead sheet of $1.3 \mathrm{~mm}$ thickness.

The photomultipliers are connected to the acquisition instrument (Instrumentation Technologies Libera BLM [18]). The input signals are sampled using an analog to digital converter (ADC) with a clock which is locked to a quarter of rf frequency, a little below $125 \mathrm{MHz}$. The ADC data are continuously monitored and counted based on the difference between the two neighboring samples. The differential mode is a feature of the acquisition system that helps to improve the counting when pileup events are observed in pulses produced by the scintillator.

Another characteristic of the acquisition system is the gating of the ADC samples. Two processing windows are generated and synchronized to the revolution clock at $533 \mathrm{kHz}$. The processing window length is set to the number of ADC samples, which means that the beam loss events are accounted for over every four bunches. The processing delay is set to receive two independent sets of ADC samples to align with the excited-not excited parts of the fill pattern.

\section{EXPERIMENTAL RESULTS}

\section{A. Continuous measurements}

The key aim of this project is to make the energy measurements compatible with the user operation using a beam with a small vertical emittance. Previously, studies at electron storage ring BESSY [3] have shown that the energy measurements were achieved during user operation. However, the vertical beam size was larger than the case of DLS. Diamond Light Source operates with a small vertical emittance in the range of $8 \mathrm{pm} \mathrm{rad}$, and the main challenge is not to affect this quantity when the beam is excited during the depolarization scans. For this reason, a low current is applied in the strip lines, which is enough to depolarize partially the beam but not to affect the quality of the beam.

One of the main challenges of the energy measurements are variations in the beam losses that are created by causes other than depolarization. To overcome this problem, the idea of gating the excitation pattern and the beam loss detection was introduced. The TMBF has the capability to select and excite only those bunches that are of interest. The beam loss acquisition system is equipped with two ADC masks which can monitor only a selected part of the beam. The main principle of the continuous measurements is to excite one part of the beam and count the beam losses using one ADC mask that is time aligned with the excited part, while the second mask counts the beam losses that are produced by another, equivalent in charge, part of the beam. The benefit of this technique is that the external factors that will modify the beam losses, like the change of the insertion device gaps, will be recorded within both masks, but the additional losses that are created due to depolarization will be seen only by one mask. As shown in Fig. 6(a), the beam loss data that are recorded from the excited and nonexcited masks during user time show the same trend until the excited mask starts to depolarize and the losses become higher. Dividing the counts from the two masks, the ratio will be equal to one in the beginning. When the depolarization happens, this ratio will change and will indicate the spin precession frequency, as shown in Fig. 6(b). The idea of the beam loss rate normalization was first introduced in storage ring VEPP-4 using the losses from two bunches circulating in the storage ring [19]. However, we established the same idea using large sets of bunches of the fill pattern independently and while preparing this paper found the earlier study at VEPP-4. The ratio between the beam loss data is fitted using an error function which calculates the energy, the width of the resonance, and the beam loss increase with the corresponding uncertainties. The increase of the ratio during the depolarization is low due to the low current that is applied in the strip lines in user time operation measurements. Nevertheless, it was sufficient for our purpose. This is a compromise in order not to affect the vertical beam size. The error of each measurement is on the order of $\mathrm{keV}$ or single-digit parts per million of the beam energy.

\section{Top-up injections}

For the online energy measurements, the excitation strength was chosen to be low in order not to affect the vertical beam size. This implies that for a decent amount of depolarization the beam should be excited for a long time according to the Froissart-Stora formula. Typically, scans last more than $10 \mathrm{~min}$, which is the time interval between the top-up events at DLS [20]. The top-up system selects 


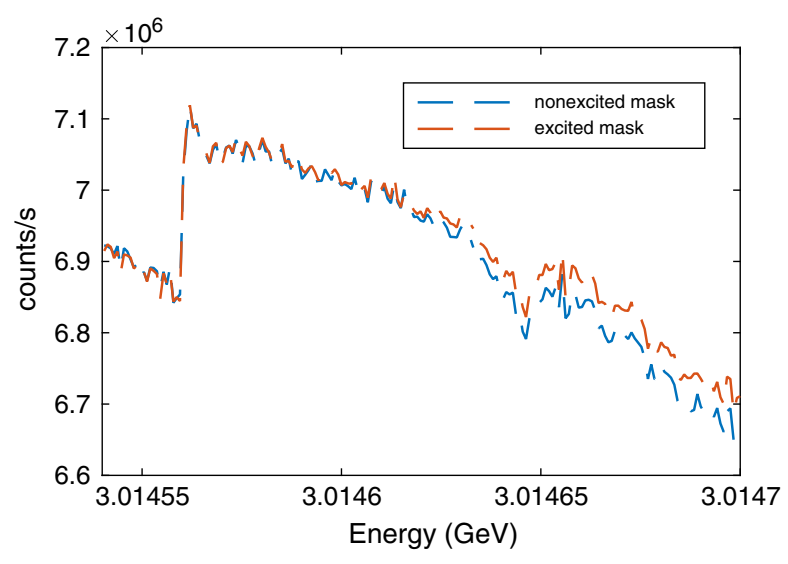

(a)

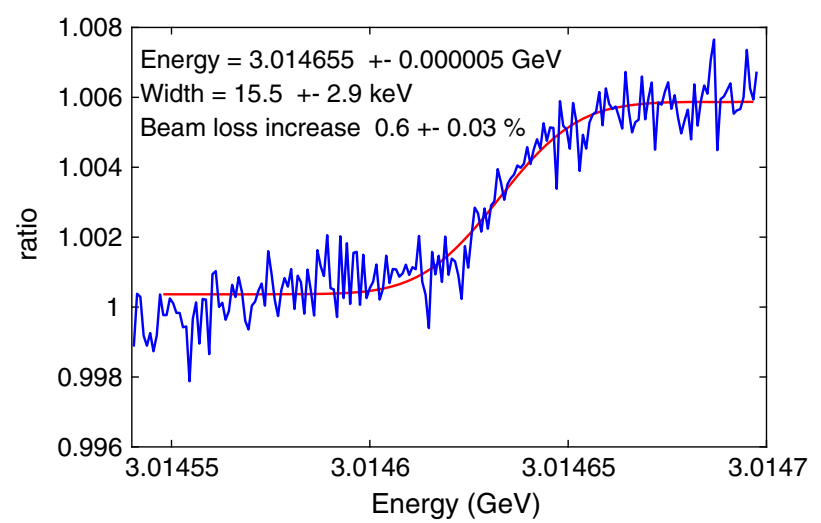

(b)

FIG. 6. The ratio of the beam loss data from the two masks show the beam depolarization.

which bunches to fill according to their charge. This creates inequalities between the loss rates of the two masks, as the loss rates depend on the total charge in each mask. For this reason, the ratio between the counts of the two masks is recorded before and after the injection without beam excitation, and the ratio is calculated and included in the analysis. Thus, we can have long scans keeping the ratio of the two masks unaffected by top-up injections.

\section{Crossing of betatron resonances}

The high sensitivity of the beam loss detectors enables them to detect losses that are created by exciting higherorder betatron resonances. During the crossing of the betatron resonances, we observe an increase in the vertical beam size accompanied by a decrease in beam losses. After the crossing, the above parameters are back to their normal values. These resonances appear as ratio changes between excited and nonexcited parts of the beam and, thus, represent additional disturbances in the measurement of beam depolarization. In Fig. 7, the red data represent the loss rates that are recorded during the time that the beam is excited. The results show two betatron resonances and an underlying depolarization resonance which is evident in the

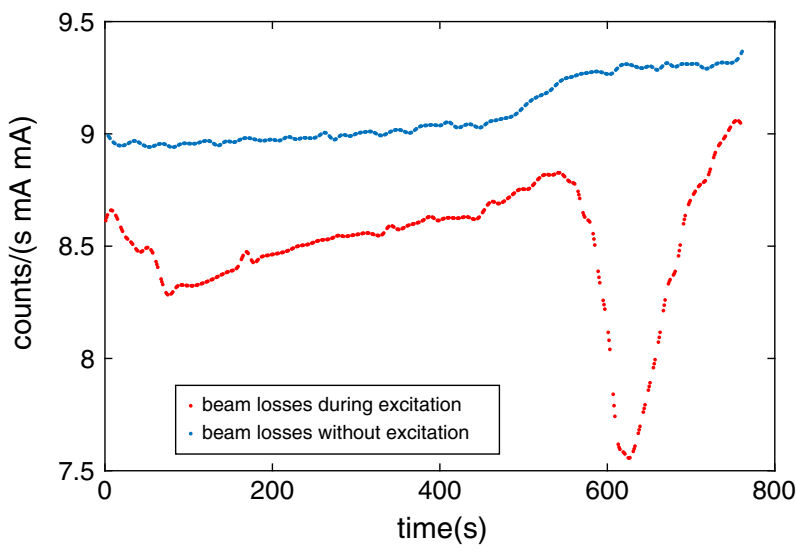

FIG. 7. The red line represent the loss rates during the excitation. The blue line corresponds to the loss rates that happen when the excitation is paused and any betatron resonances have damped out. The data are plotted versus the time to show the sequence of excitation and nonexcitation during the measurement.

increased count rate at the end of the scan. These data are difficult to be fitted, and it is impossible to calculate the energy of the beam. For this reason, we choose to switch off the depolarization signal after excitation at each frequency and record the loss rate (shown in blue in Fig. 7). Since the beam losses due to betatron resonances damp within a few milliseconds, the blue data show only the losses due to depolarization that remain. These data, which show the beam depolarization clearly, can be fitted and provide an energy measurement.

\section{B. Sidebands}

Synchrotron oscillations modulate the spin tune resulting in synchrotron sidebands around the main resonance frequency. The distance between the synchrotron sidebands and the main resonance equals the synchrotron frequency. To distinguish the main resonance from its sidebands, we deliberately vary the rf voltage, and, consequently, the synchrotron frequency changes. The rf voltage was set to 2.9, 2.5, and 2.1 MV, and the left synchrotron sideband resonance and the main resonance were swept. As is shown in Fig. 8, the left resonance was shifted $180 \mathrm{~Hz}$ toward the right in each step, which is the expected shift for a $0.4 \mathrm{MV}$ decrease in rf voltage. This measurement identifies the main resonance, which remains the same for all the scans, and the synchrotron sideband, which shifts towards the main resonance when the synchrotron frequency decreases.

For the case of $2.9 \mathrm{MV}$ rf voltage, the beam loss increase in the left sideband is lower in comparison with the other two cases. This could be caused by the incomplete equilibrium polarization, since the measurements with $2.9 \mathrm{MV}$ rf voltage started first. After the excitation of the left sideband, the ratio of the two masks remains constant as the two parts of the beam polarize simultaneously. For the other two cases, where the unexcited part of the beam has reached the maximum level of polarization, the repolarization of the excited part of 


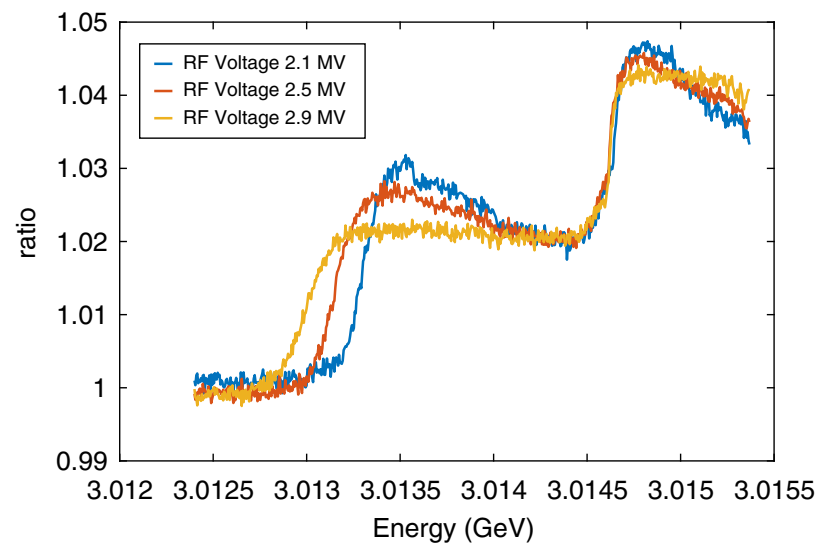

FIG. 8. Three depolarization scans to identify the main resonance and the sideband.

the beam is shown by the decrease of the beam losses until the next depolarization due to the excitation of the main resonance happens.

\section{Momentum compaction factor measurements}

The momentum compaction factor is defined as the relative change in the path length with respect to a relative change in the beam energy. Taking into account that a relative change in the rf frequency is equal and opposite to the relative change in the path length, the relationship between the rf frequency, beam momentum, and compaction factor can be defined as [21]

$$
\alpha_{c}=\frac{-\Delta f_{\mathrm{RF}} / f_{\mathrm{RF}}}{\Delta p / p}
$$

By measuring the beam energy as a function of the rf frequency, a first-order polynomial is used to extract the momentum compaction factor. For this measurement, the beam current was lowered to $50 \mathrm{~mA}$ to stay close to the model description of the storage ring. The lower loss rate due to a low beam current was not a problem for the detection of the beam depolarization due to the high sensitivity of the beam loss system. The rf frequency was varied in the range of $\pm 100 \mathrm{~Hz}$. The results are shown in Fig. 9, and the calculated value for the momentum compaction factor is $\alpha_{c}=(1.61 \pm 0.03) \times 10^{-4}$. This is close to the value calculated using the storage ring model of $\alpha_{c}=1.56 \times 10^{-4}$.

\section{DISCUSSION}

The main scope of this study is to implement the resonant spin depolarization technique during user time. The beam polarization is essential in order to conduct later the energy measurements. In the case of DLS, the maximum polarization of $88 \%$ can be achieved only when specific betatron tunes values are avoided and the beam circulates in the storage ring without the effect of magnetic field errors. In addition, during

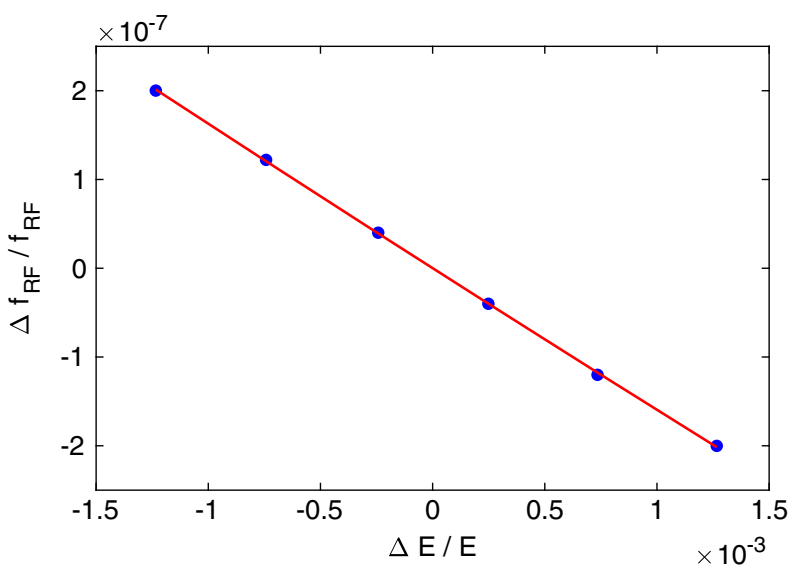

FIG. 9. The relative change in the rf frequency is plotted versus the relative change in the energy, taking into account the error of the measurement. The measured data points are shown in blue, whereas a first-order polynomial fit in red is used to extract the compaction factor in good agreement with the model.

user time, when the wigglers are turned on, the equilibrium polarization level is reduced but is enough in order to accomplish the depolarization measurements.

Regarding the depolarization measurements, the main challenge of not disturbing the vertical beam size was compensated by exciting the beam with low excitation strength and for a long time. The amount of the beam losses that are produced by this way is small, and a more advanced instrumentation system was needed for the detection of the beam losses due to depolarization. The new detectors produce a high count rate and are able to give precise readings of the beam losses. Because of the gating of the excitation and the beam loss monitor system, the normalization of the beam loss data reveals the

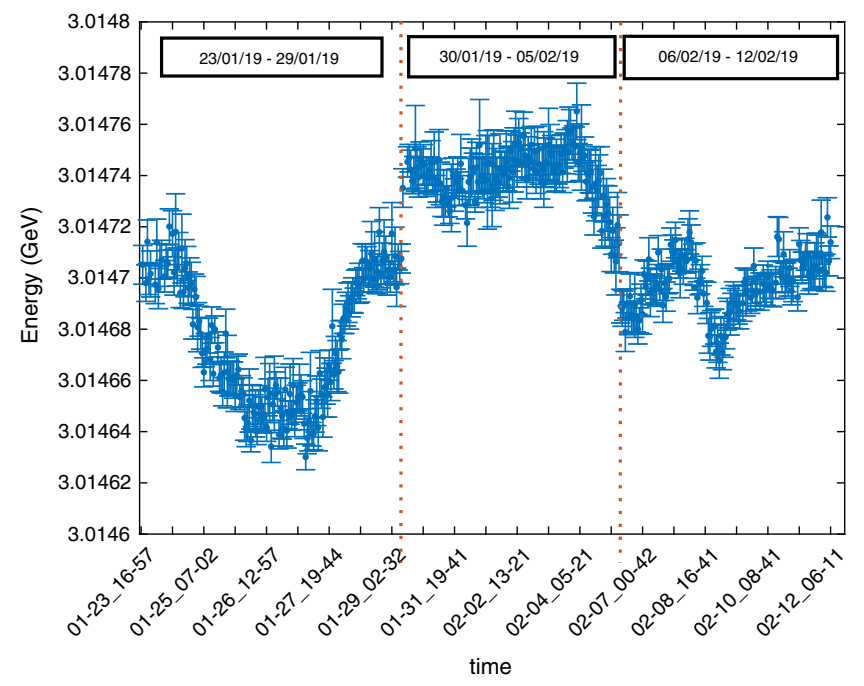

FIG. 10. Energy measurements every hour for a duration of three weeks of user operation. The energy changes over the time on the order of $10 \mathrm{keV}$. 
depolarization resonance in the presence of a significant background loss rate. These two characteristics of the instrumentation were essential for the energy measurements. An additional challenge of the measurement was the excitation of betatron resonances that overlap with the depolarization resonance. This problem was resolved by taking advantage of the fact that, when the beam excitation is stopped for one second, the beam losses due to betatron resonance will meet their normal values, but the beam losses due to depolarization will not restore. The evidence of the online measurements is shown in Fig. 10. The resonant spin depolarization scan is running continuously [22], and the energy measurements over a period of three weeks of operation are presented and show good energy stability. The small error of every measurement brings proof that the energy stability during the weeks of operation is in the range of $10 \mathrm{keV}$.

\section{CONCLUSIONS}

The level of beam polarization is critical for energy measurements using RSD. The polarization was studied theoretically and experimentally. The resonant spin depolarization technique was employed to measure the energy of the beam, and measurements of the synchrotron sidebands resonances confirmed the main depolarization resonance. The momentum compaction factor was measured using a low beam current, and the results are in good agreement with the model. A new approach of the energy measurement method with RSD was implemented in order to be compatible with the user operation mode of the storage ring, and continuous measurements were demonstrated. The challenges in implementing energy measurements during user time from the detector side to the technique were presented and novel solutions have been introduced.

\section{ACKNOWLEDGMENTS}

The authors thank J. Zhang for providing the SLIM code that was used for polarization simulations in the SOLEIL synchrotron ring and $\mathbf{J}$. Bengtsson for his useful advice on adapting the code to the diamond ring. Also, we thank Ian Martin and Richard Fielder for their inputs in this study. N. V. would like to acknowledge the support from a joint studentship between Diamond Light Source and Royal Holloway University of London (STU-184).

[1] C. Steier, J. M. Byrd, and P. Kuske, Energy calibration of the electron beam of the ALS using resonant depolarization, in Proceedings of the European Particle Accelerator Conference, Vienna, 2000, paper MOP5B03, pp. 1566-1568, https://accelconf.web.cern.ch/AccelConf/ e00/PAPERS/MOP5B03.pdf.

[2] L. Arnaudon, L. Knudsen, J. P. Koutchouk, R. Olsen, M. Placidi, R. Schmidt, M. Crozon, A. Blondel, R. Assmann, and B. Dehning (LEP Polarization Collaboration), Measurement of LEP beam energy by resonant spin depolarization, Phys. Lett. B 284, 431 (1992).

[3] P. Kuske, in Proceedings of the 5th European Workshop on Beam Diagnostics and Instrumentation for Particle Accelerators, Grenoble, France, 2001 (ESRF, Grenoble, 2001).

[4] J. Zhang, L. Cassinari, M. Labat, A. Nadji, L. S. Nadolski, and D. Pedeau, Precise beam energy measurement using resonant spin depolarization in the SOLEIL storage ring, Nucl. Instrum. Methods Phys. Res., Sect. A 697, 1 (2013).

[5] K. P. Wootton, M. J. Boland, W. J. Corbett, X. Huang, G. S. Leblanc, M. Lundin, H. P. Panopoulos, J. A. Safranek, Y. R. E. Tan, G. N. Taylor, K. Tian, and R. P. Rassool, Storage ring lattice calibration using resonant spin depolarization, Phys. Rev. Accel. Beams 16, 074001 (2013).

[6] I. Martin, M. Apollonio, R. Fielder, G. Rehm, and R. Bartolini, in Proceedings of the 2nd International Particle Accelerator Conference, San Sebastián, Spain (EPS-AG, Spain, 2011), pp. 1404-1406.

[7] J. R. Johnson, R. Prepost, D. E. Wiser, J. J. Murray, R. F. Schwitters, and C. K. Sinclair, Beam polarization measurements at the spear storage ring, Nucl. Instrum. Methods Phys. Res. 204, 261 (1983).

[8] S. R. Mane, Y. M. Shatunov, and K. Yokoya, Spinpolarized charged particle beams in high-energy accelerators, Rep. Prog. Phys. 68, 1997 (2005).

[9] M. G. Minty and F. Zimmermann, Measurement and Control of Charged Particle Beams, Particle acceleration and detection (Springer, Berlin, 2003).

[10] T. Y. Lee, J. Choi, and H. S. Kang, Simple determination of Touschek and beam-gas scattering lifetimes from a measured beam lifetime, Nucl. Instrum. Methods Phys. Res., Sect. A 554, 85 (2005).

[11] Wolfram Mathematica, https://www.wolfram.com/ mathematica/.

[12] D. P. Barber et al., High spin polarization at the HERA electron storage ring, Nucl. Instrum. Methods Phys. Res., Sect. A 338, 166 (1994).

[13] A. W. Chao, Evaluation of radiative spin polarization in an electron storage ring, Nucl. Instrum. Methods Phys. Res. 180, 29 (1981).

[14] M. Dehler, in Proceedings of the 8th European Particle Accelerator Conference, Paris, 2002 (EPS-IGA and CERN, Geneva, 2002), pp. 2070-2072.

[15] Eljen technology, http://www.eljentechnology.com.

[16] Gafchromic RTQA2 Film, http://www.gafchromic.com.

[17] N. Vitoratou, P. Karataev, and G. Rehm, Beam loss monitors for energy measurements in diamond light source, in Proceedings of International Beam Instrumentation Conference (IBIC'17) Grand Rapids, MI, USA (2017), pp. 482-486.

[18] Instrumentation Technologies, beam loss monitor readout electronics, http://www.i-tech.si.

[19] V. E. Blinov, V. O. Ivakin, V. V. Kaminskiy, V. N. Kudryavtsev, S. A. Nikitin, I. B. Nikolaev, and L. I. Shekhtman, in Proceedings of the CERN-BINP Workshop for Young Scientists in $e^{+} e^{-}$Colliders (CERN, Geneva, Switzerland, 2017), pp. 109-118. 
[20] C. Christou and V. C. Kempson, in Proceedings of the 22nd Particle Accelerator Conference, PAC-2007, Albuquerque, NM (IEEE, New York, 2007).

[21] A. Ben Kalefa, I. Birkel, E. Huttel, A. S. Muller, M. Pont, and F. Perez, in Proceedings of the 9th European Particle
Accelerator Conference, Lucerne, 2004 (EPS-AG, Lucerne, 2004), pp. 2005-2007.

[22] N. Vitoratou, P. Karataev, and G. Rehm, in Proceedings of 7th International Beam Instrumentation Conference (IBIC 2018) (Shanghai, China, 2018), pp. 107-110. 